\title{
The extended Graetz problem for micro-slit geometries; analytical coupling of rarefaction, axial conduction and viscous dissipation
}

\author{
Gulce Kalyoncu a , Murat Barisik ${ }^{\text {b, * }}$ \\ a Izmir Institute of Technology, Energy Engineering MSc. Programme, Izmir, 35430 Turkey \\ ${ }^{\mathrm{b}}$ Izmir Institute of Technology, Mechanical Engineering Department, Izmir, 35430 Turkey
}

\section{A R T I C L E I N F O}

Article history:

Received 26 November 2015

Received in revised form

10 June 2016

Accepted 20 July 2016

Available online 29 July 2016

Keywords:

Microchannel heat transfer

Slip flow

Rarefaction effect

Axial conduction

Viscous dissipation

\begin{abstract}
A B S T R A C T
In order to support the recent MEMS and Lab-on-a-chip technologies, we studied heat transport in micro-scale slit channel gas flows. Since the micro convection transport phenomena diverges from conventional macro-scale transport due to rarefaction, axial conduction and viscous heating, an accurate understanding requires a complete coupling of these effects. For such cases, we studied heat transfer in hydrodynamically developed, thermally developing gas flows in micro-slits at various flow conditions. The analytical solution of the energy equation considered both the heat conduction in the axial direction and heat dissipation of viscous forces. Furthermore, updated boundary conditions of velocity slip and temperature jump were applied based on Knudsen number of flow in order to account for the nonequilibrium gas dynamics. Local Nusselt number $(\mathrm{Nu})$ values were calculated as a function of Peclet $(\mathrm{Pe})$, Knudsen $(\mathrm{Kn})$ and Brinkman $(\mathrm{Br})$ numbers which were selected carefully according to possible micro-flow cases. Strong variation of $\mathrm{Nu}$ in thermal development length was found to dominate heat transfer behavior of micro-slits with short heating lengths for early slip flow regime. For this instance, influence of axial conduction and viscous dissipation was equally important. On the other hand, high $K n$ slip flow suppressed the axial conduction while viscous heating in a small surface-gas temperature difference case mostly determined the fully developed $N u$ and average heat transfer behavior as a function of $K n$ value.
\end{abstract}

๑) 2016 Elsevier Masson SAS. All rights reserved.

\section{Introduction}

The most recently developed small devices using micro-scale physics provide multiple advantages over their macro scale counterparts such as, lower capital costs and energy requirements, while offering higher efficiencies and simplicity in operations. However, as the size decreases, complications arise in ongoing transport behaviors. For example, the process of cooling micro electro mechanical systems (MEMS) or mixing/diffusion in Lab-Ona-Chip devices becomes more complex since the micro-level mass and heat transport displays very different behavior than conventional mechanisms. Specifically, micro gas flows diverge from Navier-Stokes solution due to the rarefaction effects. Additionally, low flow speeds and short heating lengths observed in micro flows further create axial conduction through thermal developing regions, while the large length to diameter ratios and small surface-

\footnotetext{
* Corresponding author.

E-mail address: muratbarisik@iyte.edu.tr (M. Barisik).
}

gas temperature differences build viscous dissipation; such effects are mostly neglected for macro-scales. As a result, a complete coupling of the above listed micro-scale related effects is required to understand and characterize convective gas transport of numerous existing applications and to further design new ones.

Based on current micro fabrication techniques, the manufacturing of channels is simpler than microtubes. Controlling one dimension in micro levels is the task for most cases which makes micro-slits a common geometry for multiple applications; such as microbial fuel cells [1], methanol steam reforming and hydro-energy applications [2], and evaporators for micro-power generation systems [3]. In slit geometry, characteristic flow dimensions are represented by the hydraulic diameter defined as $D_{H}=(4 A) / W$, where $A$ is the cross-sectional area and $\mathrm{W}$ is the wetted perimeter.

Gas flows in micro-geometries with small $\mathrm{D}_{\mathrm{H}}$ values undergoes non-continuum behaviors. Due to the surface-gas molecular collisions, non-equilibrium gas dynamics develops in near wall region known as Boltzmann Layer (BL). The BL extends from the surface on the order of gas mean free path $(\lambda \sim 60 \mathrm{~nm}$ for air at standard 
conditions) which is a negligible region for most conventional macro cases. As the channel dimensions gets small, BL coverage in flow domain becomes non-negligible. Simply, the ratio of BL to characteristic flow dimensions can be considered as the level of non-equilibrium gas dynamics. In Kinetic Theory, Knudsen number $\left(K n=\lambda / D_{H}\right)$ functions in such a way by calculating the ratio of the mean free path to the characteristic dimension of the flow to characterize gas rarefaction. The flow is considered as a continuum flow for small values of $K n(<0.01)$ and the well-known NavierStokes equations are applicable for the flow field. However, the decrease of characteristic length of the system increases the $K n$ which results in the continuum approach to fail. For $0.01<K n<0.1$, the flow is in slip-flow regime (slightly rarefied) where the fluid particles adjacent to the boundary surface are not in thermodynamic equilibrium with the wall, there would be slip velocity and temperature jump at the channel wall [4].

Multiple researchers implemented slip flow models successfully to consider the rarefaction effects on microscale flows [5]. Specifically, Bayazitoglu et al. [6], Rosa et al. [7], Cetin et al. [8], Cetin [9], Haddout and Lahjomri [10] and Yu and Ameel [11] observed that velocity slip increases the Nusselt number $\left(N u=\left(h \times D_{H}\right) / k\right.$, where $\mathrm{h}$ is convective and $\mathrm{k}$ is the conductive heat transfer coefficients of fluid), while the temperature jump has a negative effect on heat transfer in single phase microchannel flows.

Rarefaction is not the only complication developed in microconfinements. There are additional mechanisms which become dominant at micro-scales. The first one is the heat transfer occurring through the thermal development length, which is a major mechanism in many micro-scale heat exchangers. Originally known as the Graetz Problem, the thermal entrance region of a tube flow at a constant surface temperature was first investigated by Graetz [12] and Nusselt [13]. The authors both worked on hydrodynamically developed and thermally developing flow for a negligible axial conduction and viscous dissipation case. Since then, researchers have focused on to extension of the Graetz Problem to include additional heat transfer mechanisms, specifically for microscale flows. The second of the major micro flow effects is axial conduction. This effect especially dominates thermally developing region of heat transfer. The small Reynold numbers $\left(R e=\left(\mathrm{V} \times \mathrm{D}_{\mathrm{H}}\right)\right)$ $v$, where $\mathrm{V}$ is flow speed and $v$ is kinematic viscosity) of the microflows results in small Peclet numbers $(P e=R e \times P r$ where $P r$ is the Prandtl number); and hence, the convection term no longer dominates the conduction term in the axial direction. Analytical consideration of the Graetz Problem with axial conduction is challenging due to the resulting non-self-adjoint eigenvalues [14]. Third, the small confinements create large length to diameter ratios which develops viscous heating, and the effect of viscous dissipation becomes significant as the wall-to-fluid temperature difference becomes small in micro-convection (non-negligible Brinkman number). The viscous dissipation prolongs the thermal development through the flow direction and dominates the fully develop heat transfer.

Multiple studies exist concluding the effects of rarefaction combined with thermal development region, axial conduction and viscous dissipation for microscale flows. By adding one or two or three of the aforementioned effects, the so called extended Graetz problems were developed. Specifically, rarefaction and axial conduction for fully developed flows in microchannels studied by Hadjiconstantinou and Simek [15] where fully developed $\mathrm{Nu}$ values found to be increasing by axial conduction. Rarefaction and axial conduction were also studied for thermally developing region by Huang et al. [16], Kakac et al. [17] and Cole et al. [18] where axial conduction was found to increase the temperature at the entrance of the channel and enhance the heat transfer. Jeong and Jeong [19], Koo and Kleinstreuer [20] and Chen [21] studied viscous effects with rarefaction and reported that fully developed Nusselt number increases under viscous dissipation in the presence of small Knudsen numbers (slip flow $0.01<K n<0.1$ ). Similarly, Cetin et al. $[8,22]$ focused to extend the Graetz problem by including all three micro-scale effects. With a similar perspective, Haddout and Lahjomri [10] studied rarefaction, axial conduction and viscous dissipation effect on the Graetz problem in tube and channel geometries mostly for a case of finite heating region which is comparable with the corresponding channel height. However, there is a knowledge gap for a proper coupling of all micro-scale effects for a micro-slit convective flow. Especially, heat transfer in micro-slits at low Pe with non-negligible viscous heating through thermal development length which occasionally extends up to ten times the flow height needs to be calculated. Recently, we solved the mathematical challenge and performed a complete analytical coupling of these rarefaction, axial conduction and viscous dissipation effects for the Graetz problem in micro-tubes [23].

The objective of this study is to further derive earlier found analytical approach to devise a coupled solution for the rarefaction, viscous dissipation, and axial conduction effects on a hydrodynamically developed thermally developing micro-slit gas flow. An analytical solution will be obtained by eliminating existing mathematical challenge by using Gram-Schmidt orthogonalization accompanied with the Gauss quadrature method. Convective micro-slit gas flow in the slip-flow regime will be solved with constant wall temperature. The confluent hypergeometric functions will be employed to solve the energy equation in order to provide a fundamental understanding of the effects of the nondimensional parameters on heat transfer characteristics. Extension of thermally developing region in the axial direction is carefully analyzed at the presence of all microflow effects with various flow conditions.

\section{Analysis}

The studied geometry of the slit microchannel of hydrodynamically fully developed and thermally developing gas flow can be found in Fig. 1. The fully developed velocity profile develops through the unheated part.

For the slip flow regime, slip-velocity and temperature-jump boundary conditions were defined by Maxwell [4] as can be seen in Eqs. (1) and (2),

$$
\begin{aligned}
& u_{s}=-\frac{2-\sigma_{m}}{\sigma_{m}} \lambda\left(\frac{d u}{d y}\right)_{y @ s u r f a c e} \\
& T-T_{s}=-\frac{2-\sigma_{t}}{\sigma_{t}} \frac{2 \gamma}{\gamma+1} \frac{\lambda}{\operatorname{Pr}}\left(\frac{\partial T}{\partial y}\right)_{y @ \text { surface }}
\end{aligned}
$$

By using the slip and temperature jump boundary conditions, the non-dimensional fully developed velocity profile is obtained as follows:

$$
u^{*}=\frac{u}{u_{\text {mean }}}=\frac{3}{2} \frac{\left(1+8 K n-\left(\begin{array}{l}
y \\
H
\end{array}\right)^{2}\right)}{1+12 K n}
$$

Energy equation with the effects of viscous dissipation and axial conduction, and the boundary conditions for the flow system can be written as;

$$
u \frac{\partial T}{\partial x}=\frac{k}{\rho c_{p}}\left(\frac{\partial^{2} T}{\partial x^{2}}+\frac{\partial^{2} T}{\partial y^{2}}\right)+\frac{\gamma}{c_{p}}\left(\frac{\partial u}{\partial y}\right)^{2}
$$


Top View

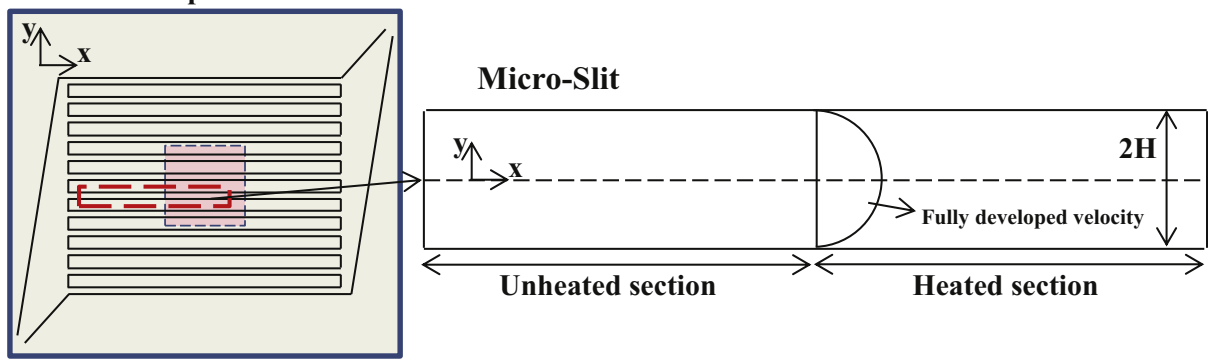

Side View

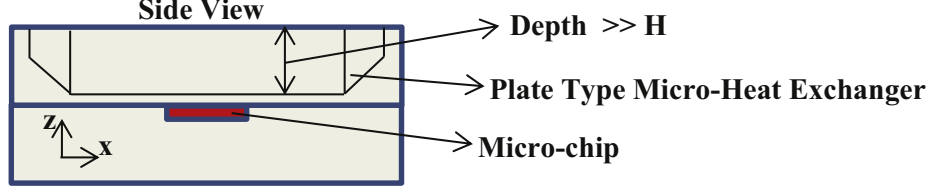

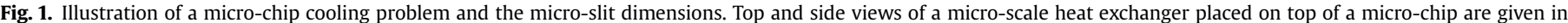
order to explain convective problem better by presenting micro-slit geometry and heating regions.

$x=0 \rightarrow T=T_{i}$

$y=0 \rightarrow \frac{\partial T}{\partial y}=0$

$\left.y=H \rightarrow T-T_{w}=-\frac{2-\sigma_{t}}{\sigma_{t}} \frac{2 \gamma}{\gamma+1} \frac{\lambda}{\operatorname{Pr}} \frac{\partial T}{\partial y}\right]_{y=H}$

Energy equation and boundary conditions are nondimensionalized by the help of following non-dimensional quantities;

$\theta=\frac{T-T_{W}}{T_{i}-T_{w}}, \quad \eta=\frac{y H}{B}, \quad \zeta=\frac{x}{H B^{3} P e} \frac{8}{3}, \quad \kappa=\frac{2-\sigma_{t}}{\sigma_{t}} \frac{2 \gamma}{\gamma+1} \frac{1}{P r}$

$u^{*}=\frac{u}{u_{\text {mean }}}, \widetilde{P e}=\operatorname{PeB}^{2} \frac{3}{8}, B r=\frac{\mu u_{m}^{2}}{k\left(T_{i}-T_{w}\right)}, A=\frac{1+8 K n}{B^{2}} B=1+12 K n$

In Eq. (5), $\kappa$ indicates the degree of temperature jump defined from Eq. (2). It is worth to note that, there is no temperature jump occurs when $\kappa$ is equal to zero. In this study, the working fluid is chosen as air since it is observed in a wide range of applications. Therefore, in the all solution procedure, $\kappa$ is used as 1.667 which is the value of air. By using these non-dimensional parameters, energy equation and boundary conditions become;

$\left(A-\eta^{2}\right) \frac{\partial \theta}{\partial \zeta^{2}}=\frac{\partial^{2} \theta}{\partial \eta^{2}}+\frac{\partial^{2} \theta}{\partial \zeta^{2}} \frac{1}{\widetilde{P e}^{2}}+B r\left(\frac{\partial u^{*}}{\partial \eta}\right)$

$\zeta=0 \rightarrow \theta=1$

$\eta=0 \rightarrow \frac{\partial \theta}{\partial \eta}=0$

$\left.\eta=\frac{1}{B} \rightarrow \theta=\frac{C_{1}}{B} \frac{\partial \theta}{\partial \eta}\right]_{\eta=1 / B}$

where $C_{1}$ is defined as;
$C_{1}= \pm \frac{2-\sigma_{t}}{\sigma_{t}} \frac{2 \gamma}{\gamma+1} \frac{4 K n}{P r}=-4 K n \kappa$

Next, $\theta(\zeta, \eta)$ can be decomposed by using superposition as,

$\theta(\zeta, \eta)=\theta_{1}(\zeta, \eta)+\theta_{\infty}(\eta)$

where $\Theta_{\infty}(\eta)$ is the fully developed temperature profile, and $\Theta_{1}(\zeta, \eta)$ is the solution of the homogeneous equation. Once Eq. (8) is substituted into the energy equation, Eq. (6a), the resulting equation becomes,

$\frac{\partial^{2} \theta_{\infty}}{\partial \eta^{2}}=-B r\left(\frac{\partial u^{*}}{\partial \eta}\right)^{2}$

The solution of Eq. (9) yields to fully developed temperature profile defined in Eq. (10);

$\theta_{\infty}(\eta)=\frac{-3 B r \eta^{4} B^{2}}{4}+\frac{12 K n \kappa B r}{B^{2}}+\frac{3}{4} \frac{B r}{B^{2}}$

Then for the homogeneous part of the temperature distribution, the following equation together with the following boundary conditions should be solved.

$\left(A-\eta^{2}\right) \frac{\partial \theta_{1}}{\partial \zeta}=\frac{\partial^{2} \theta_{1}}{\partial \eta^{2}}+\frac{\partial^{2} \theta_{1}}{\partial \zeta^{2}} \frac{1}{\widetilde{P e}^{2}}$

$\zeta=0 \rightarrow \theta_{1}=1-\theta_{\infty}$

$\eta=0 \rightarrow \frac{\partial \theta_{1}}{\partial \eta}=0$

$\left.\eta=\frac{1}{B} \rightarrow \theta_{1}=\frac{C_{1}}{B} \frac{\partial \theta_{1}}{\partial \eta}\right]_{y=1 / B}$

The solution to the boundary value problem is of the form given below [14].

$\theta_{1}(\zeta, \eta)=\sum_{n=1}^{\infty} A_{n} Y_{n}(\eta) \exp \left(-\lambda_{n}^{2} \zeta\right)$

where $A_{n}, Y_{n}, \lambda_{n}$ are the coefficients, eigenfunctions, and the 
(a)

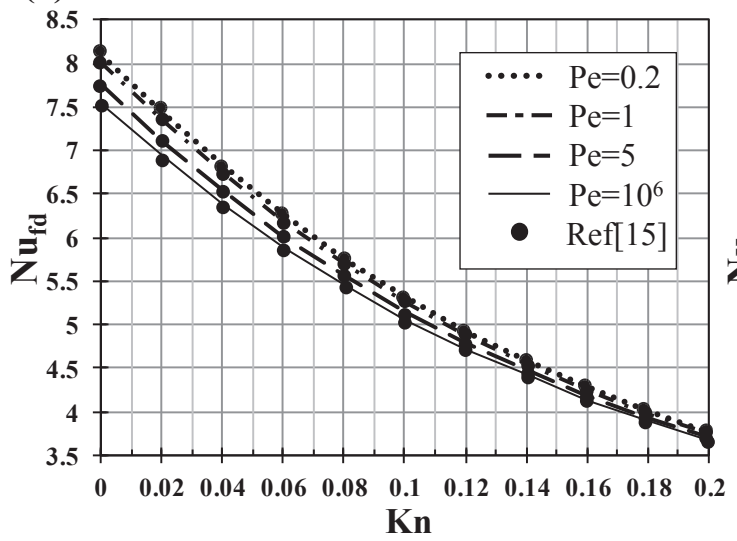

(b)

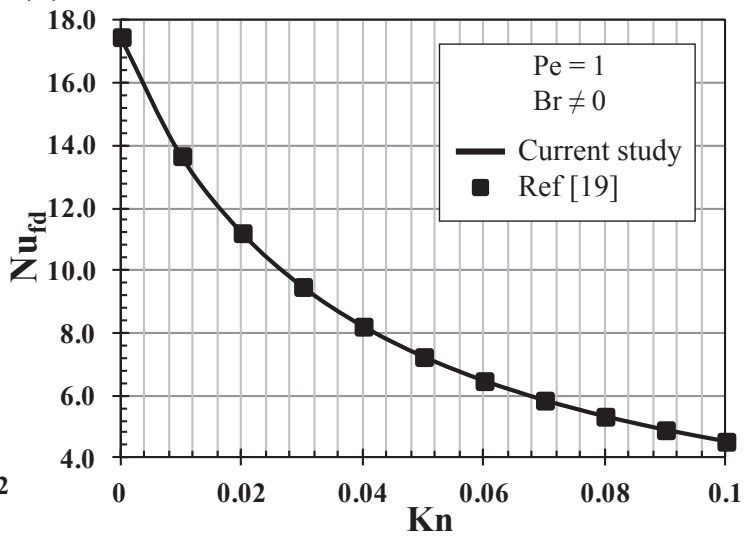

Fig. 2. Fully developed Nusselt number values as a function of Knudsen number (a) for different values of Peclet number and (b) for any Brinkman number with $\kappa=1.667$.

eigenvalues respectively. Implementation of Eq. (12) into Eq. (11a) yields to the following non-linear problem as,

$\frac{d^{2} Y_{n}(\eta)}{d \eta^{2}}+Y_{n}(\eta)\left(-\lambda_{n}^{2} \eta^{2}+A_{n} \lambda_{n}^{2}+\left(\frac{\lambda_{n}^{4}}{\widetilde{P e}^{2}}\right)\right)=0$

$\eta=0, \frac{d Y_{n}(\eta)}{d \eta}=0$

$\left.\eta=\frac{1}{B}, Y(\eta)=\frac{-4 K n \kappa}{B} \frac{\partial Y(\eta)}{\partial \eta}\right]_{y=1 / B}$

Here, the solution of Eq. (13a) is written in Eq. (14),

$Y(\eta)=1 F 1(a, c ; z) \exp \left(-\lambda \frac{\eta^{2}}{2}\right)$

The term $1 F 1(a, c ; z)$ is known as the Kummer's confluent hypergeometric function [25] and the related coefficients are calculated as;

$a=\frac{1}{4}-\frac{16 \lambda^{3}}{9 B^{4} P e^{2}}-\frac{\lambda A}{4}$ $c=1 / 2$

$z=x^{2}=\lambda \eta^{2}$

The wall boundary condition determines the eigenvalues while the summation constants can be evaluated by using the inlet boundary condition. Since the eigenvalues occur non-linearly, eigenfunctions $\mathrm{Y}(\eta)$ are not mutually orthogonal compared to the standard Sturm-Liouville problem. In order to calculate the coefficients $A_{n}$, Gram-Schmidt orthogonal procedure is used [24].

Finally, the temperature distribution $\theta(\zeta, \eta)$ is found as;

$$
\begin{aligned}
\theta(\zeta, \eta)= & \sum_{n} A_{n} 1 F 1\left(a, c ; \lambda_{n} \eta^{2}\right) \exp \left(-\lambda_{n} \frac{\eta^{2}}{2}\right) \exp \left(-\lambda_{n}^{2} \zeta\right) \\
& -\frac{3 B r \eta^{4} B^{2}}{4}+\frac{12 K n \kappa B r}{B^{2}}+\frac{3}{4} \frac{B r}{B^{2}}
\end{aligned}
$$

The bulk mean temperature and resulted Nusselt number can be calculated using Eq. (17) and Eq. (18), respectively.

$\theta_{m}(\zeta)=\int_{0}^{1} u(\eta) \theta(\zeta, \eta) d \eta$ (a)

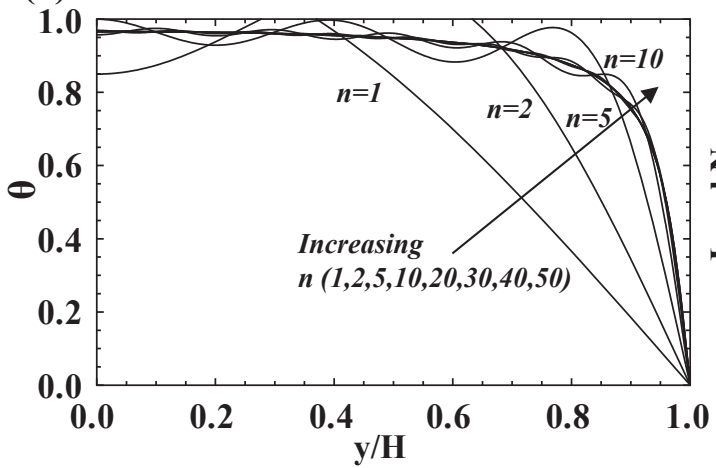

(b)

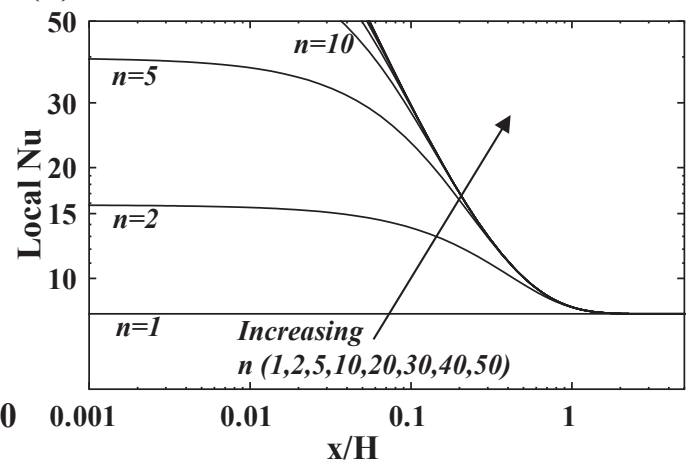

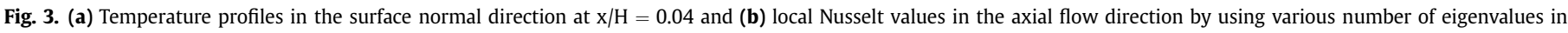
solution for $P e=1, K n=0, \mathrm{Br}=0$, and $\kappa=1.667$. 
(a)

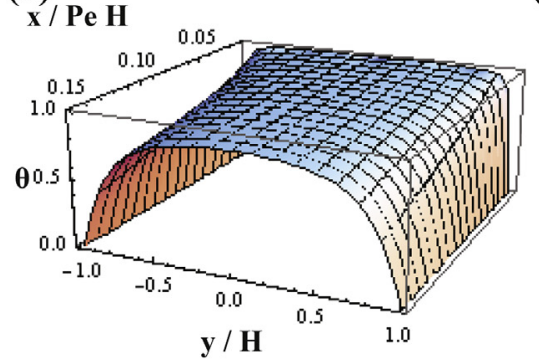

(b)

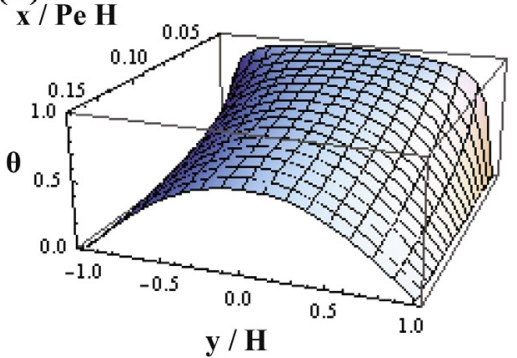

(c)

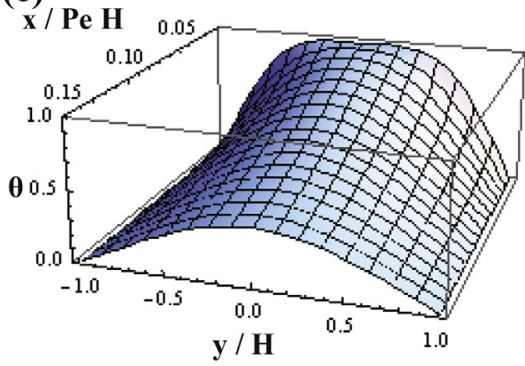

Fig. 4. Temperature profile for (a) $P e=1$ and (b) $P e=5$ and (c) $P e=50$ with $K n=0, \mathrm{Br}=0$, and $\kappa=1.667$.

$\left.N u(\zeta)=-\frac{4}{\theta_{m}(\zeta)} \frac{\partial \theta}{\partial \eta}\right]_{\eta=1}$

The solution was performed using Wolfram Mathematica software. The built in function root finder combined with a bracketing method calculated the eigenvalues. The method works up to a very high number of eigenvalues with high accuracy and short CPU times. However, for the orthogonalization part, the procedure may be complicated because of the oscillating characteristic of high order eigenfunctions. Integration of eigenfunctions results in excessive CPU time, which is not practical. This problem is eliminated by using Gaussian quadrature method for integrations. Gaussian integration with 100 points and 12 wt gives nearly the same result with direct integration in a relatively short time.

Investigations were performed as a function of non-dimensional numbers $\mathrm{Kn}, \mathrm{Pe}$, and $\mathrm{Br}$. For this case, we determined the range of these parameters based on the dimensional values observed in micro-flow cases. Assuming the air as our standard gas, we used the mean free path value of air at standard condition $(\approx 60 \mathrm{~nm})$ to determine Knudsen range. We assumed slit heights ranging between $6 \mu \mathrm{m}$ and $0.6 \mu \mathrm{m}$ which develops $K n$ range of $0.01<K n<0.1$. As the channel heights were in a possible range for micro-flows, gas velocities were also bounded based on the existing experimental gas velocity measurements for corresponding channel scales. Existing literature provides values ranging between $10 \mathrm{~m} / \mathrm{s}$ to $30 \mathrm{~m} /$ $\mathrm{s}$ for channels of $1 \mu \mathrm{m}$ to $6 \mu \mathrm{m}$ size. With the $P r=0.6$ for air, expected dimensional values yields $P e<10$ for micro gas flows. For a typical maximum temperature difference of about $60 \mathrm{~K}$ (as in, for example, electronic cooling), Brinkman number becomes $\mathrm{Br}<0.01$. Based on these calculations, $\mathrm{Br}$ range of $-0.01<\mathrm{Br}<0.01$ was selected while $P e$ values less than 50 were considered.

\section{Results and discussion}

Fully developed Nusselt number $\left(\mathrm{Nu}_{\mathrm{fd}}\right)$ values are plotted for different $K n$ numbers under the negligible and non-negligible viscous heating situations in Fig. 2(a) and (b) respectively. Overall, an increase of $K n$ increases the rarefaction which decreases the $\mathrm{Nu}_{\mathrm{fd}}$. Results were adopted from Hadjiconstantinou and Simek [15] in order to compare our findings with the solution of a molecular based method, DSMC. A good agreement observed between our analytical results and Ref [15] in Fig. 2(a). Smaller Pe values develop higher $\mathrm{Nu}_{\mathrm{fd}}$ as a result of axial conduction which becomes less prominent for high $K n$ cases. Results validates that the effect of axial conduction on $\mathrm{Nu}_{\mathrm{fd}}$ diminishes as rarefaction level increases. Viscous dissipation effect on $\mathrm{Nu}_{\mathrm{fd}}$ is presented in Fig. 2(b) for $\mathrm{Pe}=1$ flow. Although the value of both $\mathrm{Pe}$ and $\mathrm{Br}$ has significant role through the developing region, fully developed results converge to same $\mathrm{Nu}_{\mathrm{fd}}$ independent of the degree of axial conduction and viscous dissipation. It can be seen from Fig. 2(b) that the presence of viscous dissipation increases the convective heat transfer coefficient and any non-zero $\mathrm{Br}$ value increases the $\mathrm{Nu}_{\mathrm{fd}}$. Decrease of $\mathrm{Nu}_{\mathrm{fd}}$ is more profound with the increase of rarefaction for a viscous heating case. Our finding showed good agreement with Ref [19].

For the thermally developing region, accuracy of the solution mainly depends on the number of eigenvalues and eigenfunctions used in solution. Fig. 3(a) presents temperature profiles with different number of eigenfunctions in the surface normal direction, $y$, at $x=0.04$ of developing part. As the number of eigenfunctions $n$ increases, temperature profile converges to its value. Profiles with $\mathrm{n}=1,2,5,10$ and 20 cases show fluctuations through y direction while the solutions having $\mathrm{n}=30$ and higher gives the same profile. Variations of local Nusselt numbers through thermal entrance region are given in Fig. 3(b). The one eigenfunction solution can only (a)

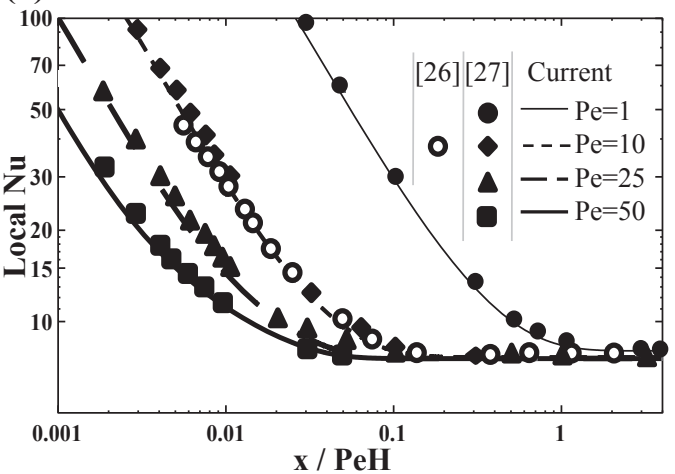

(b)

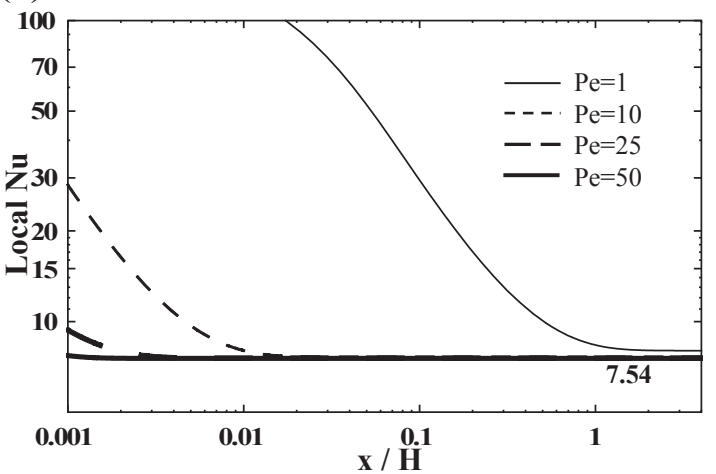

Fig. 5. Local Nusselt variation for different $P e$ values through (a) $\mathrm{x} /(\mathrm{PeH})$ and (b) $\mathrm{x} / \mathrm{H}$ at $K n=0, \mathrm{Br}=0$, and $\kappa=1.667$. Data points are adopted from Refs. [26,27]. 
(a)

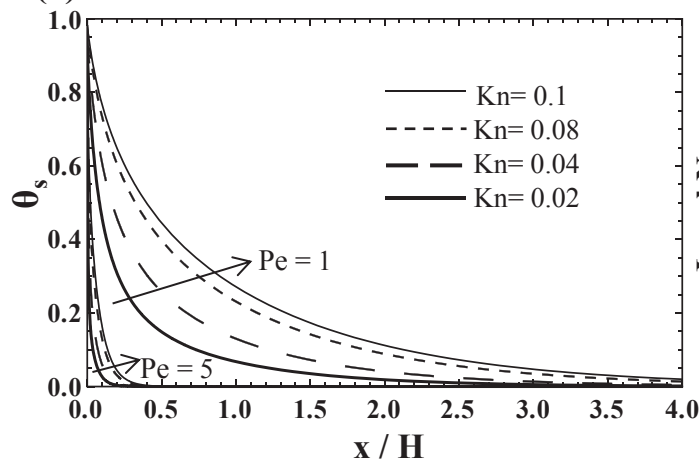

(b)

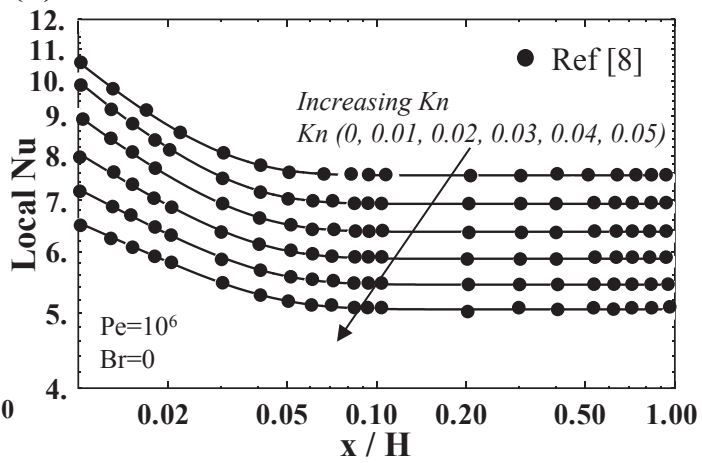

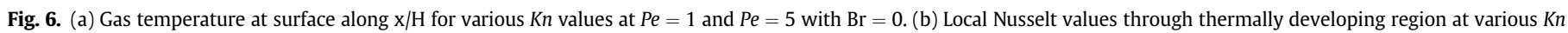
with negligible axial conduction and viscous dissipation for comparison with literature.

(a)

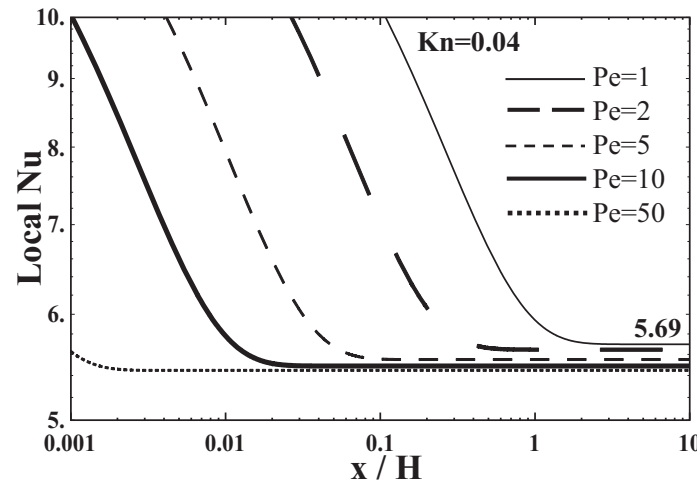

(b)

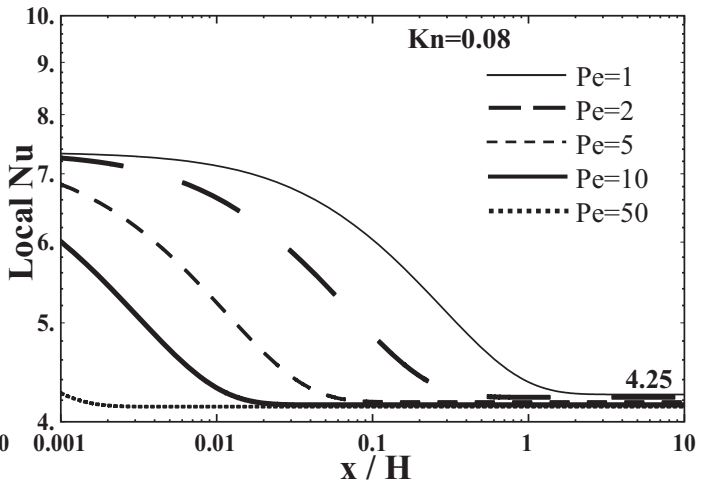

Fig. 7. Variation of local $\mathrm{Nu}$ along $\mathrm{x} / \mathrm{H}$ for different $\mathrm{Pe}$ values for (a) $\mathrm{Kn}=0.04$ and (b) 0.08 with $\mathrm{Br}=0$ and $\kappa=1.667$.

provide a constant fully develop $\mathrm{Nu}$ value, and the solutions with $\mathrm{n}=1,2,5$, and 10 also cannot resolve the behavior in the developing region. On the other hand, profiles obtained by using 30, 40 and 50 eigenfunctions were found identical. Hence, 30 eigenfunctions were accepted as the suitable number to calculate the thermally developing region.

The temperature profiles in the micro-slit for different Pe flows are given in Fig. 4 through thermally developing region. Higher temperatures are observed for low Pe values presenting the axial conduction in the system. Hence, thermal entrance length increases as the $P e$ values decrease.

Local $\mathrm{Nu}$ values of different $\mathrm{Pe}$ flows are given in Fig. 5 for $\mathrm{Kn}=0$ and $\mathrm{Br}=0$ cases. The developing region is normalized with $\mathrm{Pe}$ in addition to $\mathrm{H}$ in Fig. 5(a) in order to compare our findings with the results published in Refs. [26,27]. A good agreement is observed validating our calculations. Results show that the major impact of the axial conduction is in the developing region where much higher $\mathrm{Nu}$ values than $\mathrm{Nu}_{\mathrm{fd}}$ develop due to axial conduction. Fig. 5 also shows the development length where $\mathrm{Nu}$ reaches to $\mathrm{Nu}_{\mathrm{fd}}$. Increase of $P e$ pushes developing region towards the inlet that flow becomes fully developed earlier. Fig. 5(b) presents same results from Fig. 5(a) through a different normalization of developing region as $x / H$. The $\mathrm{x} / \mathrm{H}$ scale gives more universal normalization for different $P$ e results and can grasp the real impact of the Pe better. Fig. 5(b) reveals that flow becomes developed at $\mathrm{x} / \mathrm{H}<0.005$ for $\mathrm{Pe}>25$ values. This suggests that the effect of developing region on heat transfer becomes negligible for Pe values higher than 25 .

Temperature jump on the surface is one of the key parameter to reveal the rarefaction as a function of $K n$. Fig. 6(a) studies normalized gas temperature at the surface through the micro-slit length. The $\theta_{\mathrm{s}}$ is zero for $K n=0$ (continuum flow) as the gas temperature at surface is equal to wall temperature. However, the rarefaction of gas develops a temperature jump between the surface and adjacent gas molecules, and temperature jumps at the surface increases as the rarefaction level increases. Any finite value of $K n$ develops temperature jump between surface and adjacent gas that $\theta_{\mathrm{s}}$ gets a value between one and zero. The $\theta_{\mathrm{s}}$ values increase as the $K n$ value increase. The temperature jump is bigger at the early

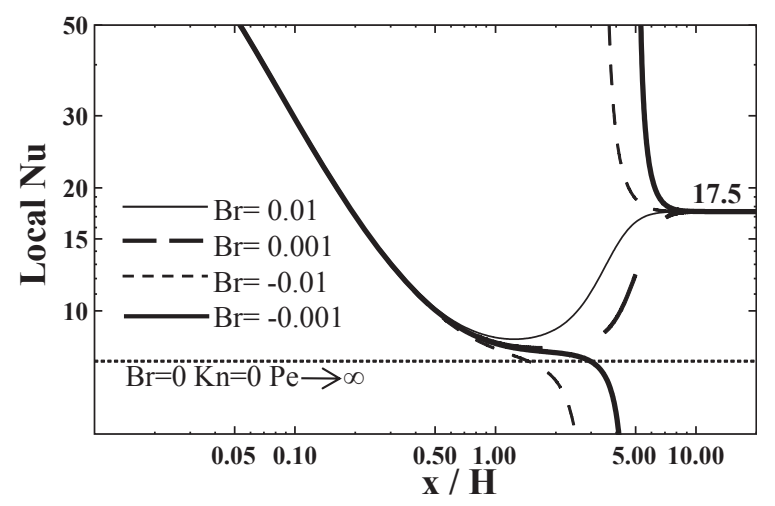

Fig. 8. Local $\mathrm{Nu}$ along $\mathrm{x} / \mathrm{H}$ in the presence of viscous dissipation with $K n=0, P e=1$, and $\kappa=1.667$. 
(a)

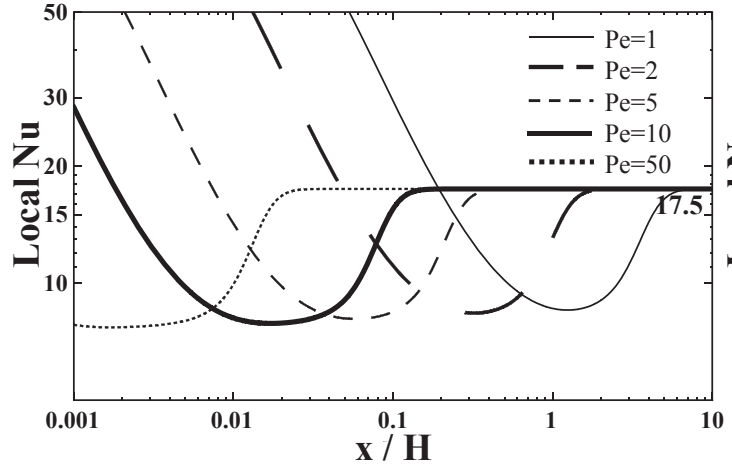

(b)

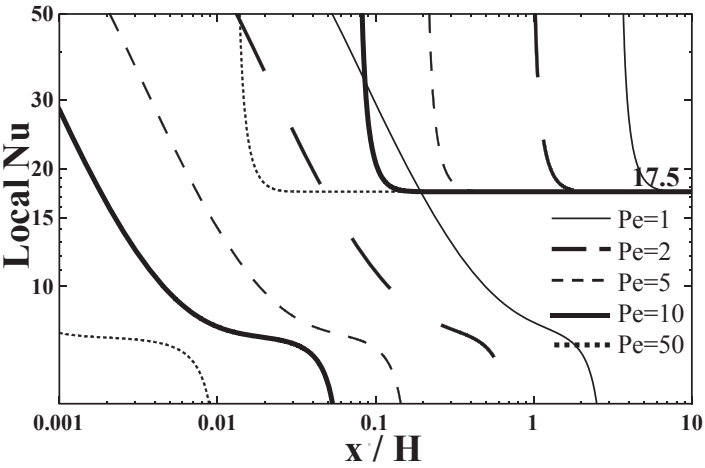

Fig. 9. Local $\mathrm{Nu}$ variation for different $\mathrm{Pe}$ values at (a) $\mathrm{Br}=0.01$ and (b) $\mathrm{Br}=-0.01$ with $\mathrm{Kn}=0$, and $\kappa=1.667$.

developing region and becomes smaller through the length. The axial conduction strongly affects this behavior that temperature jump is smaller for larger $P e$. The high axial conduction results in an increase in dimensionless temperature at any cross-section, such as at the boundaries. As a result, for low Pe values, temperature gradients and the resulting temperature jumps at the channel are stronger. Fig. 6(b) presents variation of Nusselt number through developing region at various levels of rarefaction. Results from Cetin et al. [8] were adapted in order to validate solution with nonnegligible Boltzmann layer and rarefaction.

Fig. 7 presents local $\mathrm{Nu}$ of different Pe flows at two different rarefaction levels. The axial conduction shows variation depending on the level of rarefaction. Increase of $K n$ makes axial conduction less dominant in developing region. Also, the local $\mathrm{Nu}$ values at early developing part become lower at high rarefaction which decreases the effect of developing part in the overall heat transfer.

The viscous dissipation is studied in Fig. 8 for different Br values. Similar to earlier results, fully developed $\mathrm{Nu}$ converges to same value independent to $\mathrm{Br}$ while the effect of $\mathrm{Br}$ value is observed along the developing region. Basically, local $\mathrm{Nu}$ values are the same with no viscous dissipation case of corresponding Pe flow up to some portion of $\mathrm{x}$ which determined by $\mathrm{Br}$ value. Up to this specific axial location, the effect viscous dissipation is negligible, since the axial conduction dominates early part of the thermally developing region. After this point, the viscous dissipation effect gain dominance. For such a case, viscous dissipation can be investigated in two subgroups. Positive (fluid cooling) and negative (fluid heating) $\mathrm{Br}$ numbers have different effect on local $\mathrm{Nu}$. For the case of positive $\mathrm{Br}$, viscous dissipation augments the heat transfer, increases local
$N u$. However, for negative Brinkman numbers, there forms a singularity point at which the bulk mean temperature becomes equal to the wall temperature. After this singularity point, the direction of the heat transfer changes.

The effect of axial conduction with viscous dissipation is studied in Fig. 9. It reveals that axial conduction still has a high effect through the thermally developing region up to some value of $\mathrm{x}$ depending on the $P e$ value. For $P e=1$, axial conduction determines the local $N u$ up to $\mathrm{x}=1$, after which viscous dissipation starts to influence the flow and dominates $\mathrm{Nu}_{\mathrm{x}}$. Before that location, local $\mathrm{Nu}$ variation is similar to no dissipation case. The main impact of $P e$ is on the jump point location (sudden increase of $\mathrm{Nu}$ ) in the thermally developing region. Increase in $P e$ results in the movement of jump point towards downstream. For a high $\mathrm{Pe}$ value, $\mathrm{Nu}$ reaches fully developed value at a very early part of the micro-channel.

Different levels of rarefaction in the presence of viscous dissipation are investigated in Fig. 10. The dominant effect of viscous heating is reduced as a result of velocity slip boundary condition in micro flow, and increases in rarefaction are found to decrease $\mathrm{Nu}_{\mathrm{fd}}$ values strongly. Also, the increase in $K n$ results in the transition of jump point towards upstream, similar to thermal entrance length.

\section{Conclusion}

The current study devised an analytical solution for the heat transfer problem of gas flows in micro-slit channels with a coupling of axial conduction, and viscous dissipation terms of an energy equation. Non-equilibrium in gas dynamics due to micro-scales was calculated by considering velocity slip and temperature jump (a)

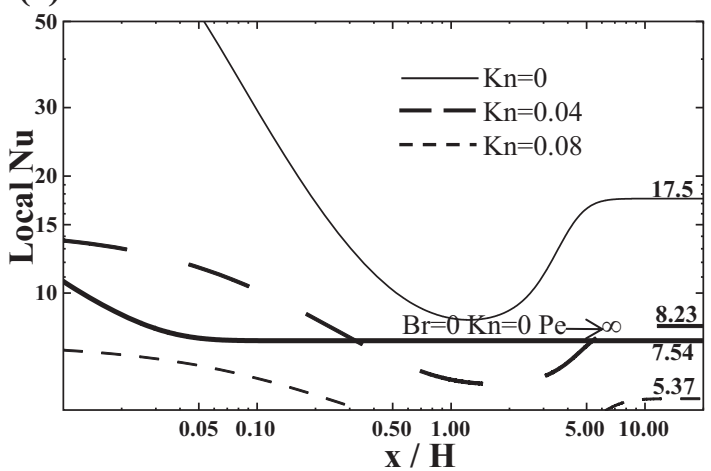

(b)

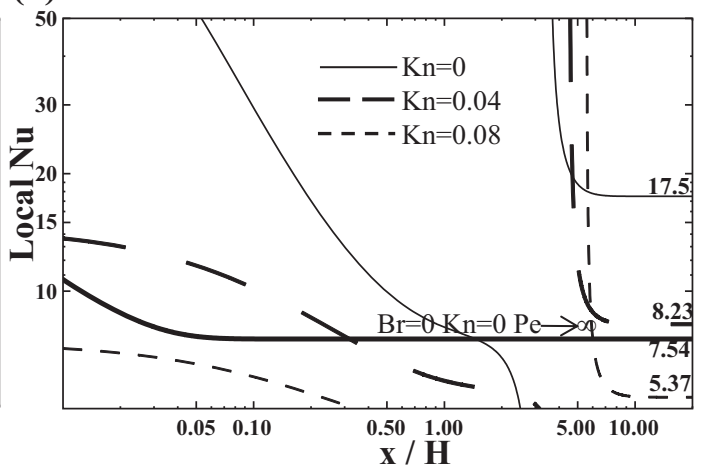

Fig. 10. Local $\mathrm{Nu}$ variation along $\mathrm{x} / \mathrm{H}$ for different $\mathrm{Kn}$ values at (a) $\mathrm{Br}=0.01$ and (b) $\mathrm{Br}=-0.01$ with $\mathrm{Pe}=1$, and $\kappa=1.667$. 
boundary conditions. Heat transfer behavior was studied as a function of non-dimensional parameters of $\mathrm{Kn}, \mathrm{Pe}$ and $\mathrm{Br}$ numbers while the studied range of these parameters was carefully determined based on existing dimensional values of micro-flow measurements and assuming air as the working fluid.

Results showed that the increase of rarefaction reduced the both local and fully developed $\mathrm{Nu}$ values. A ten times increase in $\mathrm{Kn}$ (i.e. $K n=0.01$ to $K n=0.1$ ) yield $N u$ to decrease to its half value. For a constant pressure case, a ten time increase of $K n$ can be obtained by 10 times decrease of characteristic flow dimension. We can calculate the effect of these changes on the heat transfer coefficient of $h$ as $\mathrm{h}=(\mathrm{Nu} \times \mathrm{k}) / \mathrm{D}_{\mathrm{h}}$ that decreasing flow dimensions ultimately increases the heat transfer coefficient despite the reduction of $\mathrm{Nu}$ value. This behavior validates the advantages developed due to micro-scales. While the fully developed $\mathrm{Nu}$ shows very limited effect of flow's $\mathrm{Pe}$ value, local $\mathrm{Nu}$ values undergo strong variation as a function of $P e$ which behavior is mostly confined in early thermal development region. Considering the short heating lengths and low $\mathrm{Pe}$ values for micro-convection applications, entrance regions should not be neglected and an average value of $\mathrm{Nu}$ should be calculated through the channel. On the other hand, increases in $K n$ reduce axial conduction that the high $\mathrm{Nu}$ in entrance region of low $\mathrm{Pe}$ values present a strong drop in the case of high Kn flows. For cases with viscous heating, dissipation term dominates and fully developed $\mathrm{Nu}$ converges to a value based on the $K n$ only, independent of $\mathrm{Pe}$ and $\mathrm{Br}$ values. Viscous heating enhances the heat transfer for the positive $\mathrm{Br}$ case while the negative $\mathrm{Br}$ flow experience the change of the heat transfer direction. However, both positive and negative $\mathrm{Br}$ flows develop the same $\mathrm{Nu}$ number. The influence of $\mathrm{Pe}$ and $\mathrm{Br}$ values are observed in the viscous heating case's thermal development region which is longer than the noviscous heating cases. This result validates the importance of thermal entrance region for micro-scale heat transfer problems.

\section{Acknowledgments}

Murat Barisik would like to thank The Scientific and Technological Research Council of Turkey (TUBITAK), cofunded by Marie Curie Actions under FP7 for support under the grant number TÜBITAK $115 \mathrm{C} 026$.

\section{Nomenclature}

${ }_{1} F_{1} \quad$ confluent hypergeometric function

A a non-dimensional parameter, defined in Eq. (5)

$a, c, z \quad$ Kummer's confluent hypergeometric function coefficients in Eq. (14)

$A_{n} \quad$ coefficients of eigenfunction

$B \quad$ a non-dimensional parameter, defined in Eq. (5)

$\mathrm{Br} \quad$ Brinkman number, defined in Eq. (5)

$C_{1} \quad$ coefficient in Eq. (6d), $C_{1}=-4 K n \mathrm{\kappa}$

$c_{p} \quad$ constant pressure specific heat, $\mathrm{J} / \mathrm{kgK}$

$D_{h} \quad$ hydraulic diameter $(4 \mathrm{H})$

$H \quad$ half of the channel height, $\mathrm{m}$

$k$ thermal conductivity, $\mathrm{W} / \mathrm{mK}$

$K n \quad$ Knudsen number, $K n=\lambda / D_{h}$

$\mathrm{Nu} \quad$ Nusselt number, defined in Eq. (18)

$\mathrm{Pe} \quad$ Peclet number, $\mathrm{Pe}=k /\left(\rho c_{p}\right)$

$\widetilde{P e} \quad$ Peclet number, $\mathrm{Pe}=3 B^{2} k /\left(8 \rho c_{p}\right)$

$\operatorname{Pr} \quad$ Prandtl number, $\operatorname{Pr}=\nu / \alpha$

Re Reynolds number, $\operatorname{Re}=u D_{h} / v$

$T \quad$ fluid temperature, $\mathrm{K}$

$T_{i} \quad$ inner temperature, $\mathrm{K}$

$T_{w} \quad$ surface temperature, $\mathrm{K}$

$\begin{array}{ll}u & \text { velocity in } \mathrm{x} \text { direction, } \mathrm{m} / \mathrm{s} \\ \mathrm{u}^{*} & \text { nondimensional velocity } \\ u_{s} & \text { slip velocity, m/s } \\ v & \text { velocity in y direction, } \mathrm{m} / \mathrm{s} \\ x & \text { axial coordinate } \\ y & \text { vertical coordinate } \\ Y_{n} & \text { Eigenfunction }\end{array}$

\section{Greek symbols}

$\mu \quad$ dynamic viscosity,kg/ms

$\gamma \quad$ specific heat ratio

$\zeta$ nondimensional axial coordinate, $\zeta=(8 x) /\left(3 H B^{3} \mathrm{Pe}\right)$

$\eta \quad$ nondimensional vertical coordinate, $\eta=(y H) / B$

$\theta$ dimensionless temperature, $\theta=\left(T-T_{W}\right) /\left(T_{i}-T_{w}\right)$

$\theta_{1} \quad$ solution of homogeneous equation

$\theta_{\infty} \quad$ fully developed temperature profile

$\kappa \quad$ degree of temperature jump, defined in Eq. (5)

$\lambda$ molecular mean free path, $m$

$\lambda_{n} \quad$ eigenvalues of the energy equation

$\rho \quad$ density, $\mathrm{kg} / \mathrm{m}^{3}$

$\sigma_{m} \quad$ tangential momentum accommodation coefficient

$\sigma_{t} \quad$ temperature accommodation coefficient

$v \quad$ kinematic viscosity, $\mathrm{m}^{2} / \mathrm{s}$

\section{References}

[1] Yang Y, Ye D, Li J, Zhu X, Liao Q, Zhang B. Biofilm distribution and performance of microfluidic microbial fuel cells with different microchannel geometries. Int J Hydrogen Energ 2015:1-6.

[2] Pan M, Wu Q Jiang L, Zeng D. Effect of microchannel structure on the reaction performance of methanol steam reforming. ApplEnerg 2015;154:416-27.

[3] Azarkish H, Arslan S, Behzadmehr A, Sheikholeslami TF, Sarvari SMH, Fréchette LG. Experimental and numerical investigation of a shaped microchannel evaporator for a micro Rankine cycle application. Int J Therm Sci 2015;96:191-200.

[4] Beskok A, Karniadakis GE. Simulation of heat and momentum transfer in complex micro-geometries. J Thermophys Heat Transf 1994;8:355-70.

[5] Karniadakis GE, Beskok A, Aluru N. Micro flows and nano flows: Fundamentals and simulation. New York: Springer-Verlag; 2005.

[6] Bayazıtoğlu Y, Tunc G, Wilson K, Tjahjono I. Convective heat transfer for single-phase gases in microchannel slip flow: analytical solution. In: Kakac S Vasiliev LL, Bayazitoglu Y, Yener Y, editors. Microscale heat transfer. The Netherlands: Springer; 2005. p. 125-48.

[7] Rosa P, Karayiannis TG, Collins MW. Single-phase heat transfer in microchannels: the importance of scaling effects. ApplThermEng 2009;29(No. 27): 3447-68.

[8] Cetin B, Yuncu H, Kakac S. Gaseous flow in microconduits with viscous dissipation. Int J Transp Phenom 2006;8(4):297.

[9] Çetin B. Effect of thermal creep on heat transfer for a two-dimensional microchannel flow: an analytical approach. J Heat Transf 2013;135(No. 10): 101007.

[10] Haddout Y, Lahjomri J. The extended Graetz problem for a gaseous slip flow in micropipe and parallel-plate microchannel with heating section of finite length: effects of axial conduction, viscous dissipation and pressure work. Int Heat Mass Transf 2015;80:673-87.

[11] Yu S, Ameel TA. Slip-flow heat transfer in rectangular microchannels. Int J Heat Mass Transf 2001:44(No. 22):pp.4225-4234.

[12] Graetz L. Uber die warmeleitungsfahigkeit von flussigkeiten (On the therma conductivity of liquids). Annu Rev Phys Chem Part1 1883;18:79-94.

[13] Nusselt W. Die abhangigkeit der warmeubergangszhal von der rohrlange (The dependence of the heat transfer coefficient on the tube length). VDI Z 1910;54:1154-8.

[14] Deen WM. Analysis of transport phenomena. New York: Oxford University Press; 1998. p. 387. Eq. 9.5-7.

[15] Hadjiconstantinou NG, Simek O. Constant wall temperature Nusselt number in micro and nano-channels. J Heat Transf 2002;124:356-64.

[16] Huang CY, Wu CM, Chen YN, Liou TM. The experimental investigation of axial heat conduction effect on the heat transfer analysis in microchannel flow. Int J Heat Mass Transf 2014;70:169-73.

[17] Kakaç S, Yazıcıoğlu AG, Gözükara AC. Effect of variable thermal conductivity and viscosity on single phase convective heat transfer in slip flow. Heat Mass Transf 2011;47(No. 8):879-91.

[18] Cole KD, Çetin B, Brettmann L. Microchannel heat transfer with slip flow and wall effects. J Thermophys Heat Transf 2014;28(No. 3):455-62.

[19] Jeong HE, Jeong JT. Extended Graetz problem including streamwise conduction and viscous dissipation in microchannel. Int J Heat Mass Transf 2006;49(No. 13):2151-7. 
[20] Koo J, Kleinstreuer C. Viscous dissipation effects in microtubes and microchannels. Int J Heat Mass Transf 2004;47(No. 14):3159-69.

[21] Chen C-H. Slip-flow heat transfer in microchannel with viscous dissipation. Heat Mass Transf 2006;42(No. 9):853-60.

[22] Çetin B, Zeinali S. Analysis of heat transfer for a low Peclet number microtube flow using second-order slip model: an extended-Graetz problem. J Eng Math 2014;89(1):pp.13-25.

[23] Barisik M, Yazicioglu AG, Cetin B, Kakac S. Analytical solution of thermally developing microtube heat transfer including axial conduction, viscous dissipation, and rarefaction effects. Int Commun Heat Mass 2015;67:81-5.
[24] Dutta P, Horiuchi K, Yin HM. Thermal characteristics of mixed electroosmotic and pressure-driven microflows. Comput Math Appl 2006:52(No.5):651-70.

[25] Arkfen GB, Weber HJ. Mathematical methods for physicists. New York: Academic Press; 2005.

[26] Jambal O, Shigechi T, Davaa G, Momoki S. Effects of viscous dissipation and fluid axial heat conduction on heat transfer for non-Newtonian fluids in ducts with uniform wall temperature, part I: parallel plates and circular ducts. Heat Mass Transf 2005;32:1165-73.

[27] Schmidt FW, Zeldin B. Laminar heat transfer in the entrance region of ducts. Appl Sci Res 1971;23(No. 1):73-94. 